\title{
The Effect of Polymer/ Plasticiser Ratio in Film Forming Solutions on the Properties of Chitosan Films
}

\author{
Joana F. Fundo ${ }^{1}$. Andrea C. Galvis-Sanchez ${ }^{1,2}$ • Ivonne Delgadillo ${ }^{2}$. \\ Cristina L. M. Silva ${ }^{1} \cdot$ Mafalda A. C. Quintas ${ }^{1,3}$
}

Received: 30 June 2014 / Accepted: 24 March 2015 / Published online: 8 April 2015

(C) Springer Science+Business Media New York 2015

\begin{abstract}
In this work physical-chemical properties of chitosan/ glycerol film forming solutions (FFS) and the resulting films were analysed. Solutions were prepared using different concentrations of plasticising agent (glycerol) and chitosan. Films were produced by solvent casting and equilibrated in a controlled atmosphere. FFS water activity and rheological behaviour were determined. Films water content, solubility, water vapour and oxygen permeabilities, thickness, and mechanical and thermal properties were determined. Fourier transform infrared (FTIR) spectroscopy was also used to study the chitosan/ glycerol interactions.

Results demonstrate that FFS chitosan concentration influenced solutions consistency coefficient and this was related with differences in films water retention and structure. Plasticiser addition led to an increase in films moisture content, solubility and water vapour permeability, water affinity and structural changes. Films thermomechanical properties are significantly affected by both chitosan and glycerol addition. FTIR experiments confirm these results.
\end{abstract}

This work highlights the importance of glycerol and water plasticisation in films properties.

Cristina L. M. Silva

clsilva@porto.ucp.pt

1 CBQF - Centro de Biotecnologia e Química Fina - Laboratório Associado, Escola Superior de Biotecnologia, Universidade Católica Portuguesa/Porto, Rua Arquiteto Lobão Vital, Apartado 2511, 4202401 Porto, Portugal

2 Departamento de Química, Universidade de Aveiro, Campus Universitário de Santiago, 3810-193 Aveiro, Portugal

3 IBB - Institute for Biotechnology and Bioengineering, Centre of Biological Engineering, Universidade do Minho, Braga, Portugal
Keywords Chitosan/glycerol edible films · Film forming solutions - Thermo-mechanical properties - Water content . Water activity

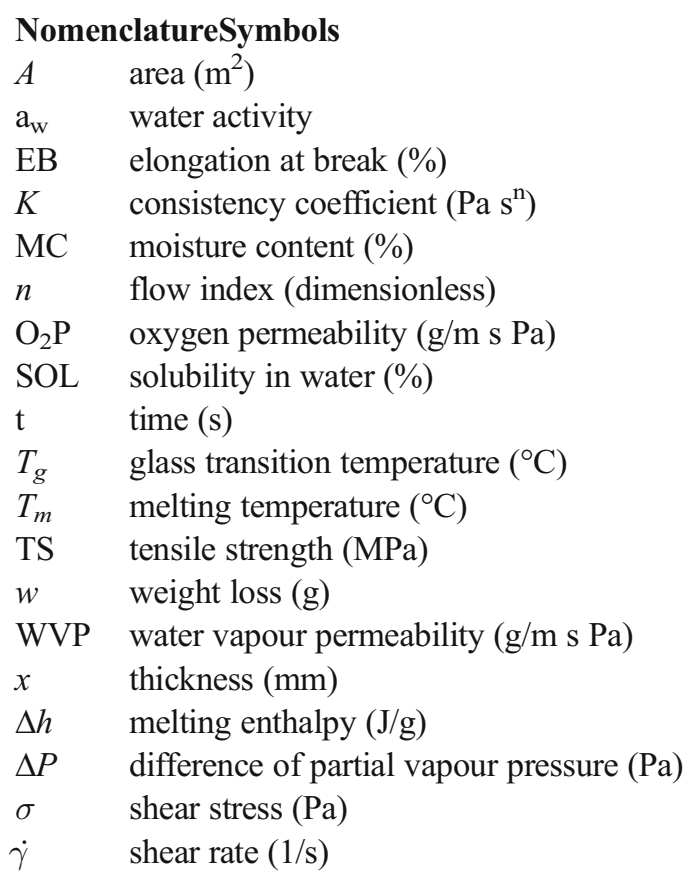

Abbreviations
A.U. arbitrary units
Chit chitosan
FFS film forming solutions
Gly glycerol

\section{Introduction}

Edible films have been successfully explored at experimental level, attracting interest in the food preservation field, mainly 
aiming at application of coatings to perishable foods. The literature is extensive in characterisation of such materials, and particularly in reporting the thermo mechanical behaviour and barrier properties of glassy biopolymers and polymers [1,2].

However, films technology still presents several challenges, especially on the relationship between the composition and properties of film forming solutions and the properties of the obtained films. This may be of particular importance for those films which are produced by solvent casting for research/ development, and are mainly intended to be applicable to food products at industrial level by immersion (or spraying) and air drying of the solution. The viscosity and molecular entanglement of the film forming solutions are then of great importance since it may affect the obtained film properties such as thickness, mechanical and thermal properties, water retention capacity, water affinity and $\mathrm{O}_{2}$ permeability.

Natural polymers are inherently brittle due to their complex branched primary structure and weak intermolecular forces [3]. The primary role of plasticisers is to improve the flexibility and processability of polymers, by reducing the intermolecular forces, softening the rigidity of the film structure and rising the mobility of the biopolymeric chains [3, 4]. These additives reduce the tension of deformation, hardness, density, viscosity and electrostatic charge of a polymer, and at the same time increase chain flexibility, resistance and dielectric constant [5].

Glycerol is the mostly used plasticiser due to its good efficiency, large availability and low exudation [6]. Glycerol has also been used to modify natural macromolecules like proteins $[7,8]$ and carbohydrates.

Water is also one of the most important plasticisers of biological systems, such as foods $[9,10]$, since water molecules create hydrogen bonds with the polymeric chains present in the system. Thus, water influences the biopolymers physical properties, e.g., relaxation [11].

Chitosan is a semicrystalline biopolymer, having a great potential for chemical and mechanical modifications to create novel properties, functions and applications in different areas [12]. This polymer is biocompatible, biodegradable and possesses antimicrobial activity and excellent filmogenic properties $[13,14]$. Due to its properties, the use of chitosan in edible films development has been long studied $[10,12]$. These studies have shown that chitosan films properties depend on several parameters, such as chitosan molecular weight and degree of deacetylation, organic acid used and the possible presence of plasticiser $[6,15]$.

The structure of the film is strongly affected by the composition, specially the amounts of polymer and plasticiser, in the film forming solution and the ratio between these compounds, and is one of the main responsible for its properties. This is reported to be related with the polymer free volume, which affects molecular mobility of the polymeric matrix $[16,17]$.
This paper aims at systematically investigating how the properties and structure of chitosan films are influenced by the properties and composition of the film forming solutions. To achieve that, film forming solutions were prepared with 3 different chitosan levels and with 3 chitosan/glycerol ratios, and the rheological behaviour was characterised. The water, barrier, mechanical and thermal properties and FTIR spectra of the obtained films were characterised.

\section{Materials and Methods}

\section{Chitosan Film Forming Solutions (FFSs) Preparation}

FFSs were prepared by dissolving different chitosan $(90 \%$ deacetylation, Aqua Premier Co., Thailand) concentrations $(1 \%, 2 \%$ and $3 \% \mathrm{w} / \mathrm{v})$ in a $1 \%$ lactic acid solution (Acros Organics, Belgium), with 3 different levels (10\%, $50 \%$ and $90 \% w / w)$ of plasticising agent, (glycerol - Panreac, Spain). These conditions correspond to always the same ratio chitosan/ glycerol (see Table 1). It was decided not to consider films with $0 \%$ of glycerol, since these films are too brittle, making impossible to perform most of the analysis. To promote a good homogenisation an Ultra-Turrax was used (IKA T18 basic, Wilmington, NC, USA). To allow significant comparisons, two replicates (samples) were made for each experimental condition (chitosan/ plasticiser ratio).

\section{Characterisation of FFSs}

\section{Rheological Behaviour}

Rheology of FFSs was studied by viscometry tests, using a controlled stress rheometer Bohlin VOR (Bohlin Instruments Ltd, Cirencester UK) at $23^{\circ} \mathrm{C}$ and a cone-plate configuration. For each sample three measurements were carried out.

\section{Water Activity}

Measurements were performed with a dew point hygrometer (Aqualab - Series 3, Decagon Devices Inc., USA.), at $23 \pm$ $1{ }^{\circ} \mathrm{C}$. The sensitivity of the equipment was 0.001 . Calibration was carried out before experiments with distilled water and saturated saline solutions. Water activity $\left(\mathrm{a}_{\mathrm{w}}\right)$ value of each sample was the average of nine readings.

\section{Chitosan Films Preparation}

A constant amount $(300 \mathrm{ml})$ of the chitosan solutions was casted in $32 \times 40 \mathrm{~cm}$ plates and dried in an incubator at $40{ }^{\circ} \mathrm{C}$, for 3 days. Prior to any characterisation, films were stored at $22{ }^{\circ} \mathrm{C}$ and $53 \% \mathrm{RH}$, until equilibrium was reached. Once again, to allow significant comparisons, two replicates 
Table 1 Experimental results for the characterisation of FFS

\begin{tabular}{|c|c|c|c|c|c|c|c|}
\hline \multirow[b]{2}{*}{$\begin{array}{l}\text { Chit } \\
(w / v \%)\end{array}$} & \multirow[b]{2}{*}{$\begin{array}{l}\text { Gly } \\
(w / v \%)\end{array}$} & \multirow[b]{2}{*}{$\begin{array}{l}\text { Chit/ Gly } \\
\text { Ratio }(w / w)\end{array}$} & \multicolumn{2}{|l|}{ Viscosity Parameters } & \multirow[b]{2}{*}{$\begin{array}{l}a_{w} \\
( \pm \text { Standard Deviation })\end{array}$} & \multirow[b]{2}{*}{$\begin{array}{l}\text { Gly } \\
*\end{array}$} & \multirow[b]{2}{*}{$\begin{array}{l}\text { Chi } \\
*\end{array}$} \\
\hline & & & $\begin{array}{l}K\left(\mathrm{~Pa} \mathrm{~s}^{\mathrm{n}}\right) \\
( \pm 95 \% \text { Confidence Error })\end{array}$ & $\begin{array}{l}n \\
( \pm 95 \% \text { Confidence Error })\end{array}$ & & & \\
\hline 1 & 10 & 7.92 & $0.198 \pm 0.010$ & $0.810 \pm 0.018$ & $1.002 \pm 0.001$ & a & a \\
\hline 1 & 50 & 1.59 & $0.254 \pm 0.021$ & $0.803 \pm 0.006$ & $1.000 \pm 0.001$ & a & $\mathrm{a}$ \\
\hline 1 & 90 & 0.88 & $0.219 \pm 0.013$ & $0.814 \pm 0.004$ & $0.999 \pm 0.002$ & a & a \\
\hline 2 & 10 & 7.94 & $2.132 \pm 0.430$ & $0.656 \pm 0.018$ & $1.001 \pm 0.001$ & a & $\mathrm{a}$ \\
\hline 2 & 50 & 1.58 & $1.450 \pm 0.104$ & $0.682 \pm 0.001$ & $1.001 \pm 0.001$ & $\mathrm{a}$ & $\mathrm{a}$ \\
\hline 2 & 90 & 0.88 & $1.591 \pm 0.135$ & $0.683 \pm 0.008$ & $0.997 \pm 0.001$ & a & a \\
\hline 3 & 10 & 7.94 & $3.371 \pm 0.260$ & $0.620 \pm 0.005$ & $1.002 \pm 0.001$ & $\mathrm{a}$ & $\mathrm{a}$ \\
\hline 3 & 50 & 1.59 & $3.221 \pm 0.095$ & $0.623 \pm 0.003$ & $0.999 \pm 0.001$ & $\mathrm{ab}$ & a \\
\hline 3 & 90 & 0.88 & $3.034 \pm 0.130$ & $0.635 \pm 0.002$ & $0.995 \pm 0.002$ & $\mathrm{~b}$ & $\mathrm{a}$ \\
\hline
\end{tabular}

*Mean values followed by the same letter are not significantly different at 0.05 by the Tukey HSD test; Letters from Gly column concern to differences between glycerol concentrations (for the same chitosan concentration); Letters from Chit column refer to analysis of the effect of chitosan concentration (for the same glycerol content)

of films (samples) were produced for each experimental condition (chitosan/ plasticiser ratio). All measurements were done in a controlled temperature $\left(22{ }^{\circ} \mathrm{C}\right)$ and humidity $(53 \%)$ room.

\section{Characterisation of Chitosan Films}

\section{Films Water Activity, Moisture Content and Solubility in Water}

Films water activity $\left(\mathrm{a}_{\mathrm{w}}\right)$ was determined using the same methodology described under point Water Activity.

To determine the films moisture content (MC), approximately $50 \mathrm{mg}$ of film were dried at $105^{\circ} \mathrm{C}$, until weight equilibrium was attained. The weight loss of the sample was determined, and $\mathrm{MC}$ was calculated as the percentage of water removed from the system. Three measurements were obtained for each sample..

Solubility (SOL) was determined as the content of dry matter solubilised after $24 \mathrm{~h}$ of immersion in distilled water. Two pieces of each sample, previously dried until constant weight, were immersed in $50 \mathrm{~mL}$ of water (at $23{ }^{\circ} \mathrm{C}$ ). After $24 \mathrm{~h}$ of immersion with agitation, the pieces of film were taken out and dried until constant weight in an oven at $105{ }^{\circ} \mathrm{C}$, to determine the weight of dry matter not solubilised in water. Solubility of films in water was determined as the percentage of soluble material (SOL). Three measurements were obtained for each sample.

\section{Films Barrier Properties}

Water vapour permeability (WVP) was evaluated gravimetrically based on ASTM E96-92 method [18-20]. The film was sealed on the top of a permeation cell containing distilled water $(100 \% \mathrm{RH} ; 2337 \mathrm{~Pa}$ vapour pressure at room temperature), placed in a desiccator at $22{ }^{\circ} \mathrm{C}$ and $0 \% \mathrm{RH}(0 \mathrm{~Pa}$ water vapour pressure) containing silica. The cells were weighed at intervals of $2 \mathrm{~h}$ for $10 \mathrm{~h}$ using an analytical balance [18]. Two measurements were made for each sample.

Oxygen permeability $\left(\mathrm{O}_{2} \mathrm{P}\right)$ was determined based on the ASTM D 3985-02 (2002) method [21]. Briefly, the films were sealed between two chambers, having each one two channels. In the lower chamber, $\mathrm{O}_{2}$ was supplied at a controlled flow rate (J \& W Scientific, ADM 2000, USA) to maintain its pressure constant in that compartment. The other chamber was purged by a stream of nitrogen, also at controlled flow rate. Nitrogen acted as a carrier for the $\mathrm{O}_{2}$. To determine $\mathrm{O}_{2}$ concentration, $1 \mathrm{~mL}$ of sample was injected in a gas chromatograph (Chrompack 9001, Middelburg, The Netherlands) at $110^{\circ} \mathrm{C}$ with a column Porapak Q 80/ 100 mesh $2 \mathrm{~m} \times 1 / 8^{\prime \prime} \times$ $2 \mathrm{~mm} \mathrm{SS}$, using a thermal conductivity detector (TCD) at $110{ }^{\circ} \mathrm{C}$. Helium at $23 \mathrm{~mL} \cdot \mathrm{min}^{-1}$ was used as carrier gas. A standard mixture containing $10 \% \mathrm{CO}_{2}, 20 \% \mathrm{O}_{2}$ and $70 \% \mathrm{~N}_{2}$ was used for calibration. The flows of the two chambers were connected to a manometer to ensure the equality of pressures (both at $1 \mathrm{~atm}$ ) between both compartments. As the $O_{2}$ was carried continuously by the nitrogen flow, it was considered that partial pressure of $\mathrm{O}_{2}$ in the upper compartment is null, therefore $\Delta P$ is equal to $1 \mathrm{~atm}$. Three measurements were taken for each sample.

\section{Film Thickness}

The thickness of the produced films was measured using a digital micrometer (Mitutoyo, Japan). From each sample a minimum of 8 stripes $(15 \times 170 \mathrm{~mm})$ were cut, and at least 2 readings were randomly taken at different positions. 


\section{Films Mechanical Properties}

Films mechanical properties, elongation at break (EB) and tensile strength (TS), were determined in extension with an Instron Universal Testing Machine (Model 4500, Instron Corporation, U.S.A.), following the guidelines of ASTM D 882-91 (1991). The initial grip separation and the crosshead speed were set at $100 \mathrm{~mm}$ and $50 \mathrm{~mm} \mathrm{~min}^{-1}$, respectively. EB was calculated as the ratio of the final length at the point of sample rupture to the initial length of a specimen $(100 \mathrm{~mm})$, and expressed as a percentage. TS was expressed in MPa and calculated dividing the maximum load $(\mathrm{N})$ by the initial crosssectional area of the specimen. EB and TS tests were replicated nine times for each sample.

\section{Films Thermal Properties}

Films thermal profiles, glass transition temperature $\left(T_{g}\right)$, melting enthalpy $(\Delta h)$ and melting temperature $\left(T_{m}\right)$, were determined using differential scanning calorimetry (DSC). DSC was performed by a TA-60WS, Shimadzu Corporation, Japan, with a cooling accessory, under $N_{2}$ atmosphere $(20 \mathrm{~mL} / \mathrm{min})$.

Film samples of approximately $5 \mathrm{mg}$ were weighed into aluminium cups and sealed hermetically. An empty cup was used as reference and the temperature was increased at $20^{\circ} \mathrm{C} /$ min, from -150 to $200{ }^{\circ} \mathrm{C}$. The maximum temperature of $200^{\circ} \mathrm{C}$ was selected in order to limit possible chitosan degradation [20]. Thermograms were analysed using the Universal Analyses Software TA-60WS (Shimadzu Corporation, Japan). Two measurements were made for each sample.

\section{FTIR-ATR Spectroscopy}

All spectra were acquired using a spectrometer Perkin-Elmer (Spectrum BX) set up for mid-infrared measurements equipped with a horizontal one single reflection ATR Golden Gate (Specac, Germany). The software OPUS v. 5.0 (Brüker, Germany) was programmed to record each spectrum between 4000 and $600 \mathrm{~cm}^{-1}$ at a resolution of $4 \mathrm{~cm}^{-1}$. Samples and background measurements were made by coadding 128 scans for each spectrum before Fourier transformation. The interferometer was operated at a laser frequency of $10 \mathrm{kHz}$ and in the single-sided directional mode. Fourier transformation was done with a Mertz phase correction, a Triangular apodisation function, with a zero-filling factor of 2. At least three spectrum replicates were recorded for each film composition.

\section{Data Analysis}

To conclude on the isolated effect of chitosan addition in film forming solutions and obtained films $(p<0.05)$, experimental results were analysed by one-way ANOVA and post hoc multiple comparison tests (Tukey's test), for a fixed glycerol concentration. To evaluate glycerol addition, statistical analysis of the data was performed fixing the chitosan concentration.

To assess samples rheological behaviour a power law model (Eq. 1) was fitted to the experimental data of shear stress $(\sigma)$ as a function of shear rate $(\dot{\gamma})$ :

$\sigma=k(\dot{\gamma})^{n}$

were $n$ is the flow behaviour index, and $K$ the consistency coefficient.

WVP was estimated using regression analysis from Eq. (2); adapted from literature [22] and corresponding $95 \%$ confidence intervals were calculated:

$\frac{w \times x}{A \times \Delta P}=\mathrm{WVP} \times t$

where $x$ is the average thickness of edible films, $A$ the permeation area $\left(0.005524 \mathrm{~m}^{2}\right), \Delta P$ the difference of partial vapour pressure of the atmosphere $\left(2337 \mathrm{~Pa}\right.$ at $\left.20^{\circ} \mathrm{C}\right), w$ the weight loss, and $t$ the experimental time.

Spectra analysis was performed using the program CATS 97 [23]. Principal component analysis (PCA) was used for reducing the dimensionality of the data and to extract the main sources of variability.

\section{Results and Discussion}

\section{Characterisation of the FFSs}

The rheological behaviour and the $a_{w}$ of the FFSs used in this study are presented in Table 1 .

For all the tested FFSs, results show a shear thinning behaviour, which is commonly used for describing the polymer melt behaviour [24]. The Power Law Model (Eq. 1) successfully described the obtained rheograms. $n$ and $K[25,26]$ were estimated and the corresponding $95 \%$ confidence limits calculated (Table 1).

Glycerol addition showed no significant effect on FFS rheological behaviour, assessed by $n$ and $K$ estimates. On the other hand, chitosan concentration, affected significantly FFS rheological behaviour, ranging from close to Newtonian (low chitosan concentrations, $n \rightarrow 1$ ) to a pseudoplastic behaviour (with increasing chitosan concentration, $n$ decreasing). Also, $K$ significantly increased with chitosan addition. These results can be related with the lower amounts of water present in the solutions with higher polymer/plasticiser concentration.

As discussed above, although different chitosan and glycerol concentrations were used to prepare the FFS, for each chitosan level studied $(1,2$ and $3 \%)$ the same ratios chitosan/ glycerol were tested (see Table 1). However, the 
observed rheological behaviour showed that the amount of polymer in the solution has a higher impact on viscoelastic properties of the FFS than the ratio between polymer and plasticiser.

Regarding $a_{w}$, a significant effect of chitosan and glycerol addition was observed, while no differences were found between replicates ( $p>0.05$, Main Effects ANOVA, results not shown). To conclude on the significance $(p<0.05)$ of the isolated effect of glycerol concentration on the $a_{w}$ of the FFS (for the same chitosan concentration), results were analysed using the glycerol concentration as the categorical predictor factor (Gly column on Table 1). For testing significance of the effect of chitosan concentration (for the same glycerol concentration), results were analysed using chitosan concentration as the factor (Chit column on Table 1).

Results show that there is no significant effect of the amount of the polymer present on the $a_{w}$ of the solutions. However, the addition of glycerol as plasticiser showed a different effect depending on chitosan concentration: for lower chitosan concentration ( $1 \%$ ), the addition of glycerol did not affect $a_{w}$. However, with increasing chitosan concentration, glycerol addition decreases solutions $a_{w}$. This effect is evident for $3 \%$ chitosan concentration. Statistical analyses show that solutions with $3 \%$ chitosan concentration and different levels of glycerol are significantly different, while at lower chitosan concentration (1\%) glycerol additions lead to no significant differences between samples (Gly column on Table 1).

These results may indicate that interaction of the plasticiser and of the water molecules with the polymeric chain plays a critical role not only in films, but also on the FFSs and may influence the water evaporation during films drying.

\section{Characterisation of Chitosan Films}

\section{Water and Barrier Properties}

Experimental results for $a_{w}$, MC, SOL and WVP (water related properties) of chitosan films are shown in Table 2. No differences between replicates were observed ( $p>0.05$, Main Effects ANOVA, results not shown). Again, to conclude on the significance of glycerol and chitosan concentrations effects on the different film's properties, experimental results were analysed first using the glycerol concentration as categorical predictor (Gly column on Table 2). For testing significance of the effect of chitosan concentration (for the same glycerol concentration), results were analysed using chitosan concentration as the factor (Chit column on Table 2).

Water activity results show that chitosan has a significant effect on this parameter (Table 2). However, glycerol only has a significant effect for films produced with higher chitosan content $(3 \%)$. In this case, higher glycerol content led to higher $a_{w}$ values. These results may be related with the polymer, plasticiser and/ or water ratios and bindings.

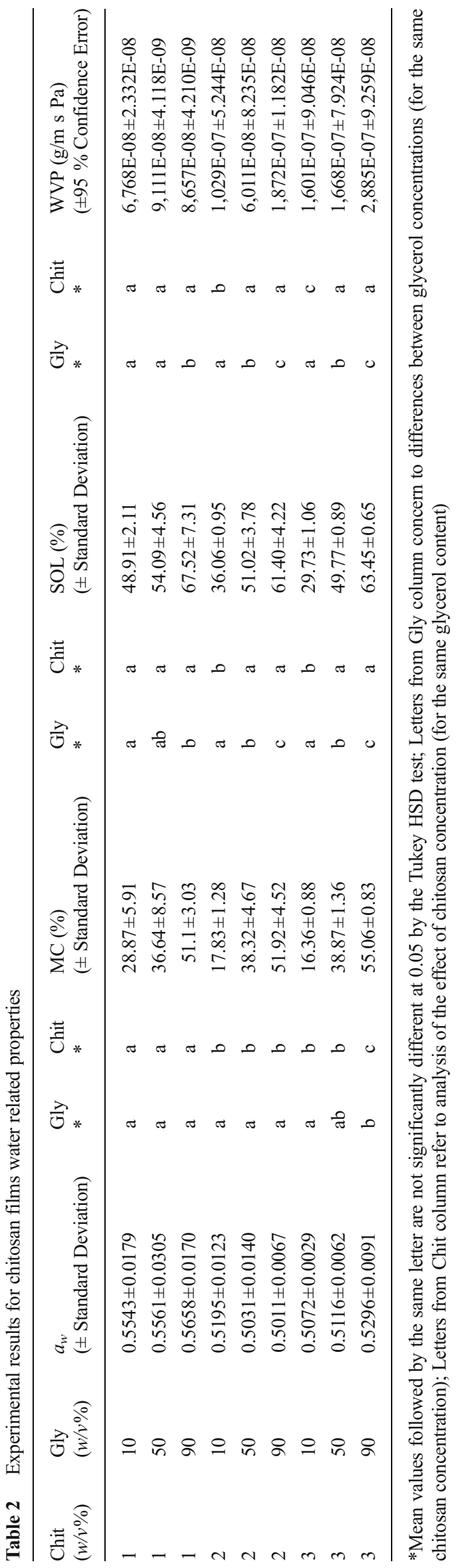


Regarding MC, it was observed that higher glycerol concentration solutions produce films with significantly higher MC. For every chitosan concentration, increasing plasticiser content on the FFS produced a significant increase of film's MC (Table 2).

SOL was significantly higher in films produced with higher glycerol concentrations (Table 2). However, chitosan content only had a significant effect on solubility of films produced with low glycerol content (10\%). These results may be related with high solubility of glycerol in water (and its hygroscopic nature), due to the three hydrophilic hydroxyl groups present [26].

For the results of chitosan films WVP it is observed that there are significant differences for different chitosan concentrations (Table 2): higher chitosan concentrations led to higher values of WVP. This tendency could be explained by an increase of amino groups present and consequent higher hydrophilicity of the biodegradable blend films when increasing the chitosan content [27]. Also, in most of the films analysed, samples with higher plasticiser concentration show higher WVP values. These results are probably due to an increase in the free volume between the polymer chains - when hydrophilic plasticisers are incorporated into polysaccharide films there is a decrease of the intermolecular forces, making the polymer network less dense and hence more permeable [28, 29].

With respect to samples $\mathrm{O}_{2} \mathrm{P}$ no significant differences nor between chitosan neither between glycerol concentrations were observed (results not shown). Nevertheless, Fig. 1 demonstrates a general tendency with respect to polymer and plasticiser proportions: higher chitosan concentrations in the FFSs led to higher values of $\mathrm{O}_{2} \mathrm{P}$; and higher plasticiser concentrations led to lower values of $\mathrm{O}_{2}$ permeability. The exception is samples produced with $1 \%$ of chitosan, where $\mathrm{O}_{2} \mathrm{P}$ values are almost constant for different glycerol concentrations. This may indicate structural changes that should be investigated in the future, and are supported also by a direct

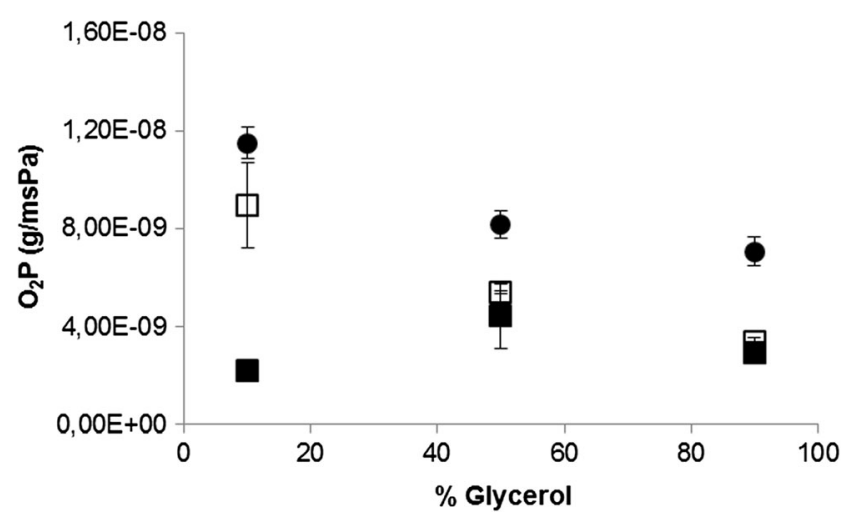

Fig. $1 \mathrm{O}_{2} \mathrm{P}$ of films prepared with different chitosan and glycerol concentrations (black square $1 \%$, white square $2 \%$; black circle $3 \%$ chitosan). Bars mean the standard error relationship between decreasing crystallinity of the films (see Table 3 on the section below) and the decrease in $\mathrm{O}_{2} \mathrm{P}$.

Overall, the addition of plasticiser led to an increase in MC, SOL and WVP of the films, showing increased water affinity and structural changes. This was also reflected on $\mathrm{O}_{2} \mathrm{P}$ decrease with glycerol addition. Chitosan concentration did not affect significantly such properties.

\section{Mechanical and Thermal Properties}

The experimental results for the mechanical and thermal analysis of chitosan films are presented in Table 3.

The films thickness was only significantly affected by the chitosan content, showing that possible structural changes due to plasticisation e.g., increase in free volume, are not reflected on this property. Also, the MC (Table 2) showed no relationship with the thickness of the obtained film. This may indicate that chitosan is the main contributor to film thickness.

Regarding the films mechanical properties, both chitosan and glycerol addition led to significant differences in EB and TS. At $1 \%$ chitosan, the amount of glycerol added shows a conventional action of plasticisers, increasing EB and decreasing the TS. This effect is due to chitosan chains interactions, decreasing intermolecular attraction and increasing polymer mobility, which facilitates film elongation $[15,30]$. However, films produced with solutions with higher chitosan content (2 and 3 $w / v \%)$ and $50 \%$ of glycerol had a deviant behaviour: showing higher EB than films with $90 \%$ of glycerol. This behaviour has been previously observed and may occur due to the relationship between polymer/ plasticiser concentrations, corresponding to an antiplasticisation phenomenon: A stronger interaction might be occurring between the polymer and the plasticiser, producing a "cross-linker" effect, which decreases the free volume and the molecular mobility of the polymer [15, 30, 31].

$T_{g}$ is associated with a change in the physical properties and state of materials, and can be related with the plasticisation of amorphous regions within semi-crystalline materials [32]. $T_{g}$ is considered a second order phase transition and occurs over the temperature range at which a glassy material enters the rubbery domain [2]. At temperatures above $T_{g}$ various physical properties are significantly affected $[2,16]$.

Table 3 shows the $T_{g}$ for the different films in this study. Results demonstrated that glycerol significantly affects films $T_{g}$. As was expected, plasticiser (glycerol) lowered $T_{g}[15$, 33], which also correlated well with moisture content (Table 2), since water acts as plasticiser itself [33-35]. The chitosan/ glycerol ratio also affected $T_{g}$ (Fig. 2a): increasing ratio lead to a $T_{g}$ increase. This may be related with the free volume in the films: as was discussed above. Higher plasticiser content increases free volume and higher polymer content decreases this variable [31-33].

The crystalline component of the films was evaluated by the $\Delta h$ and $T_{m}$. Table 3 shows that $\Delta h$ increased with increasing 


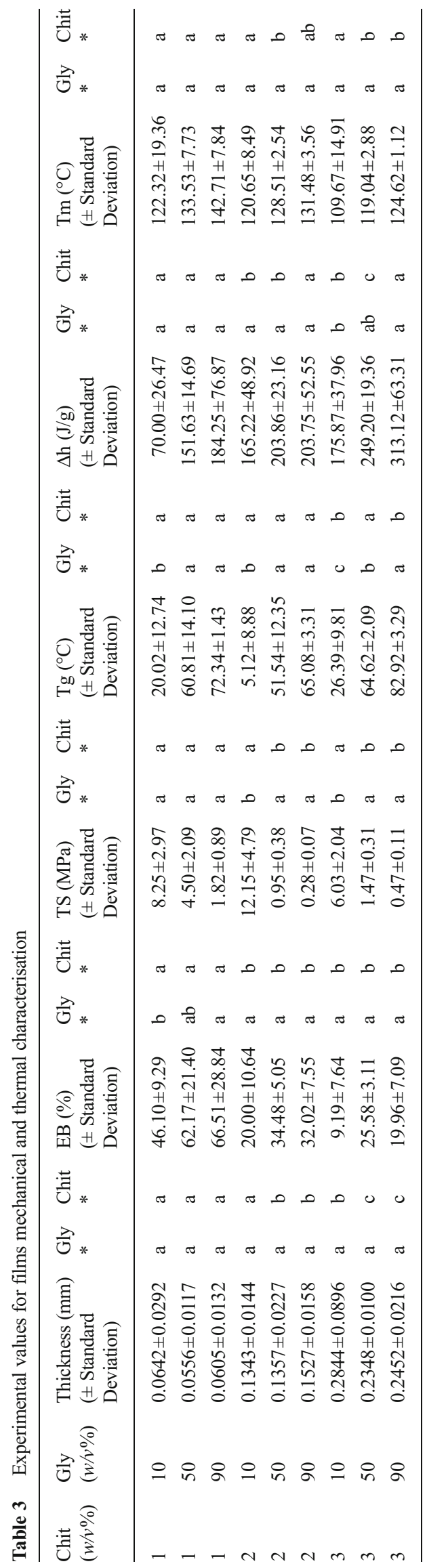

chitosan concentrations, particularly for samples produced with lower glycerol concentrations. This result was expected once chitosan, as a polymer, is responsible for the formation of crystals in the system. Also, plasticiser addition increased the melting enthalpy, presenting significant differences for formulations produced with $3 \%$ of chitosan, i.e., increases the samples crystallinity, and this may be due to glycerol interaction with chitosan chains: the H-bonds stabilise the chitosan crystals [36].

In Fig. $2 \mathrm{~b}$ the values of the $\mathrm{Tm}$ are at around 110 to $140{ }^{\circ} \mathrm{C}$. Despite these values were not significantly different between formulations, a tendency in values depending on the FFSs composition is observed. Higher concentrations of chitosan present lower $T m$ values. On the other hand, temperature of the main peak shifted to higher melting temperatures when increasing plasticiser concentration (decreasing ratio). This correlated well with published results [33] and may be also related with an increase of the strength of the H-bonds stabilising the chitosan crystals in the presence of plasticiser [36].

Overall, thermal and mechanical characterisation showed a significant effect on the properties of films produced with FFSs of different compositions. Once again, the observed effect on these properties reflects changes in the films structure.

\section{FTIR-ATR Spectroscopy Results}

FTIR has been extensively applied for the characterisation of biopolymers as this technique reveals specific information about the molecular structure of chemical compounds [37, 38]. Also, important information about specific interactions between the different constituents of the biopolymers can be extracted from the infrared spectrum.

Figure 3 presents the results obtained from FTIR measurements. Figure 3a presents typical spectra of the different films with different compositions. In order to better understand the possible interaction of the different constituents of the films, a band assignment was performed by comparing the films' spectrum with the spectra of their pure compounds and comparison with literature results.

The residual lactic acid is evident in all films, as confirmed by a band arising at $1715 \mathrm{~cm}^{-1}$ (Fig. 3a) corresponding to the $\mathrm{C}=\mathrm{O}$ from the carboxylic acid stretching [38].

Analysis of the whole spectra shows differences in the regions between 3400 and $2815 \mathrm{~cm}^{-1}$, refining the band with increasing glycerol content. This region corresponds to the stretching vibrations of the $-\mathrm{O}-\mathrm{H}$ and $-\mathrm{C}-\mathrm{H}$ groups, present in glycerol $\left(\mathrm{C}_{3} \mathrm{H}_{8} \mathrm{O}_{3}\right)$.

Literature reports that chitosan with $85 \%$ of degree of deacetylation displayed two strong vibration bands at 1645 and $1584 \mathrm{~cm}^{-1}$; those bands were assigned to amide I and amide II vibrations, respectively. It is also reported that amine deformation vibrations usually produce strong to very strong bands in the $1638-1575 \mathrm{~cm}^{-1}$ region [38]. 
Fig. $2 T_{g}(\mathbf{a})$ and $T_{m}(\mathbf{b})$ of the chitosan films prepared with different chitosan and glycerol concentrations (black square $1 \%$, white square $2 \%$; black circle $3 \%$ chitosan)

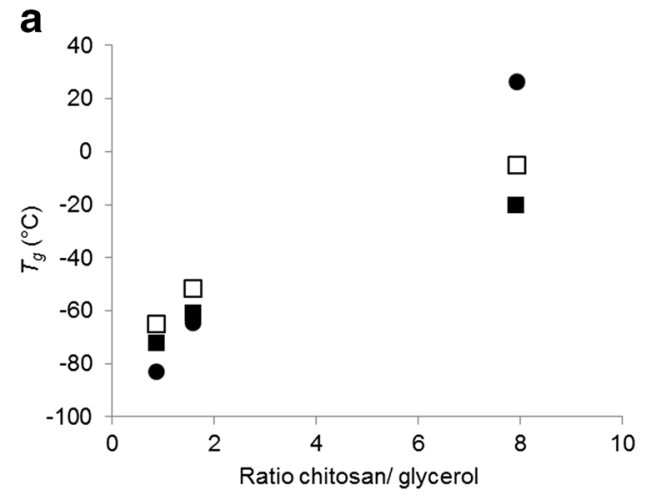

In the chitosan spectrum bands arising at 1638 and $1583 \mathrm{~cm}^{-1}$ are seen, corresponding to the amide I, amine II and to the amine deformation (results not shown). In Fig. 3a a
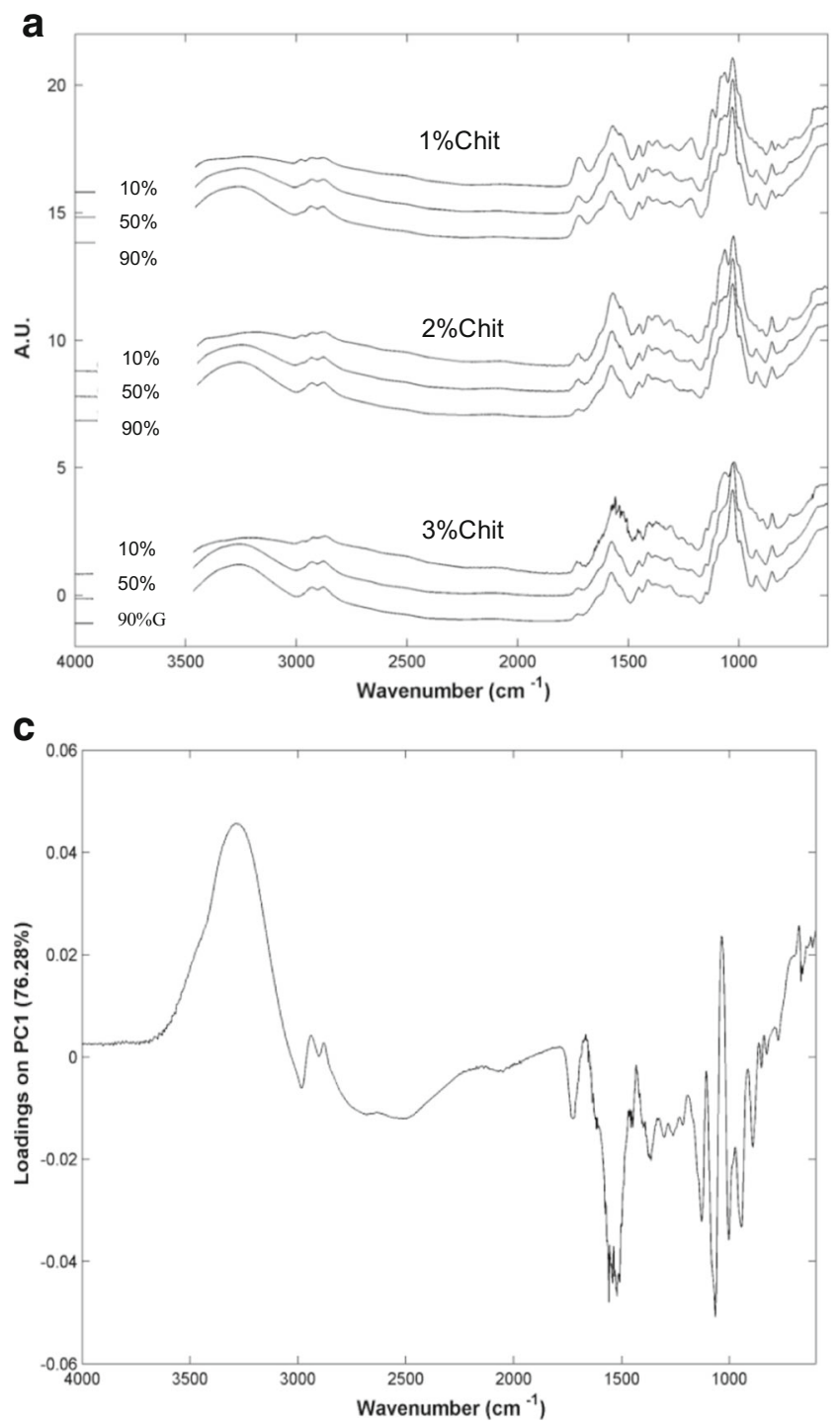

Fig. 3 Results obtained from FTIR measurements: a) FTIR measurements with different chitosan/ glycerol percentage, b) representation of the scores resulting from PCA model applied to the films with

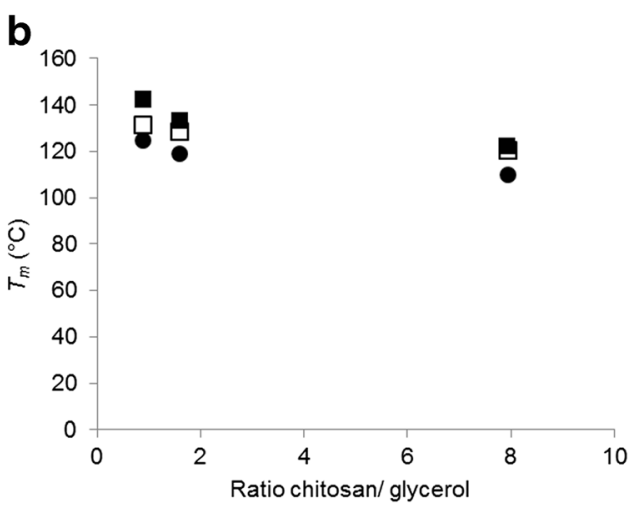

big band at $1569 \mathrm{~cm}^{-1}$ in between of two shoulders at 1631 and $1529 \mathrm{~cm}^{-1}$ are observed. The shift of those bands when compared with the pure chitosan spectrum could be due to the

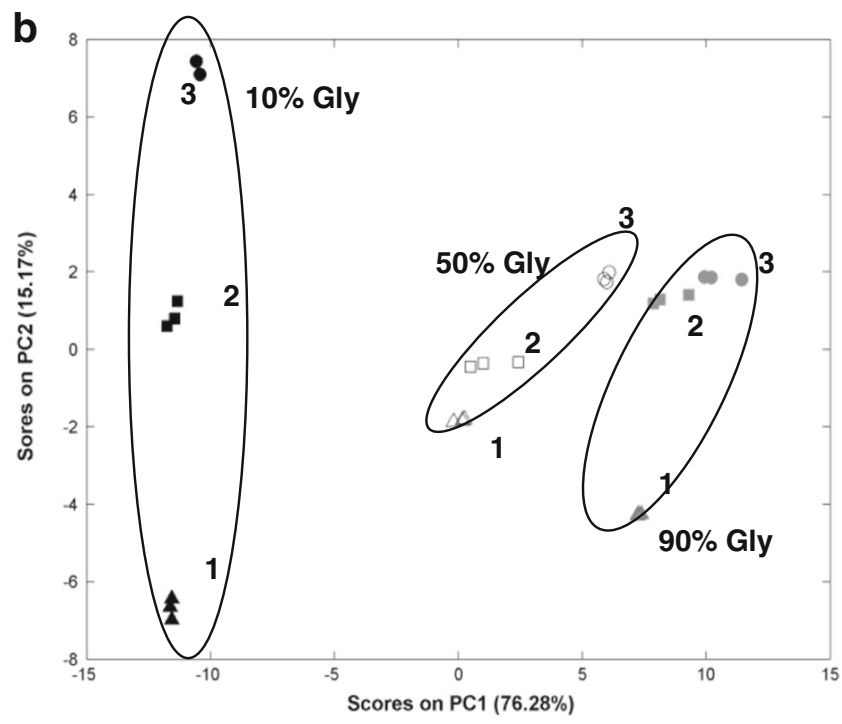

d

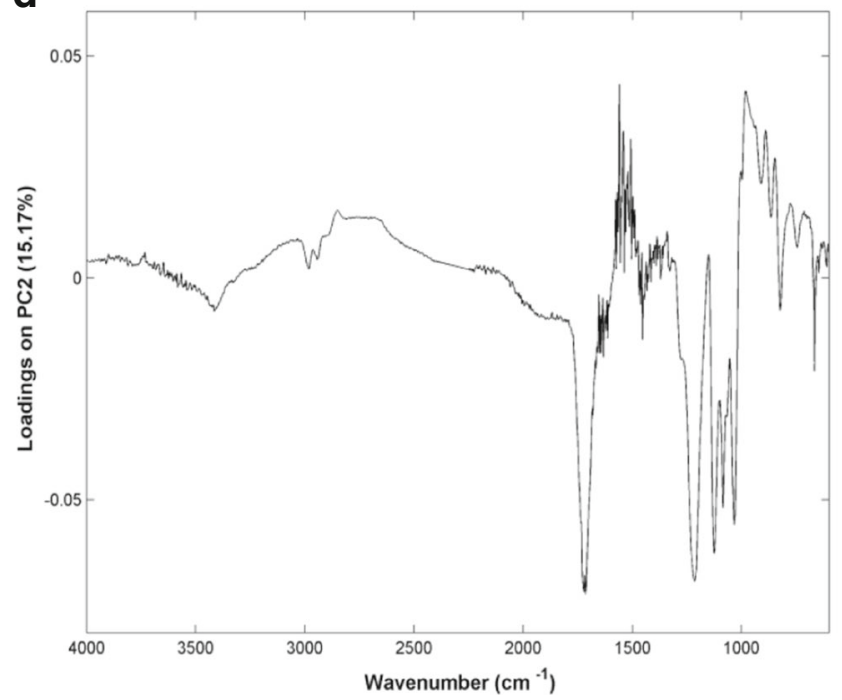

different chitosan/glycerol percentages (1- 1, 2- 2 and 3-3\% of chitosan) and c) PC1 and d) PC2 loadings profile plots of films according their chitosan/ glycerol composition 
NH bending vibration at $1583 \mathrm{~cm}^{-1}$, which overlaps the amide II. Also, considering the protonation of the amines which can cause an antisymmetric deformation in the $1625-1560 \mathrm{~cm}^{-1}$ range and a symmetric deformation in the $1550-1505 \mathrm{~cm}^{-1}$ range and the amide and amine moieties present in the films, the two represented bands must embody an envelope of at least five bands in close proximity [38].

Previous works have observed that the intensity of the amide II band was significantly affected by the level of plasticiser in a protein based film - films without glycerol presented a broader band's shape when compared with those with $40 \%$ of plasticiser in its composition [37]. This observation is in agreement with the results presented in Fig. 3a, where films with lower concentration of glycerol $(10 \%)$ in their composition presented a untidy band at $1569 \mathrm{~cm}^{-1}$ when compared with the films with higher plasticiser ( 50 and $90 \%$ of glycerol) in their composition. This indicates higher molecular vibration in the films with higher plasticiser content, which may be correlated by an increase of molecular mobility in these samples. Such hypothesis is supported by the increase of crystallinity in these samples (Table 3), with consequent increased free volume.

Figure $3 \mathrm{~b}$ represents the PCA analysis of the films with different compositions; this figure confirms the previous results showing that the films form three homogenous clusters along the PC1 (reflecting glycerol interaction).

The loading profile of $\mathrm{PC} 1$ (Fig. 3c) shows that the separation in the 3 different clusters is due mainly to the $-\mathrm{O}-\mathrm{H}$ stretching vibration at $3265 \mathrm{~cm}^{-1}$, the $-\mathrm{O}-\mathrm{H}$ bending at $1665 \mathrm{~cm}^{-1}$, the vibrations of $-\mathrm{C}-\mathrm{H}$ group at $1433 \mathrm{~cm}^{-1}$, reflecting the increased glycerol and water content of the films, and to the C-O stretch vibrations with bands between 1300 and $1000 \mathrm{~cm}^{-1}$ range, reflecting differences in the interaction between the different components (chitosan/glycerol/ residual lactic acid) depending on film forming solution composition. Figure $3 \mathrm{~d}$ also shows the separation of the films along the PC2 - in this case the contribution for this separation was attributed to the chitosan interaction, which increased with FFSs chitosan concentration.

It is important to notice that the separation of samples within the same cluster decreased as the proportion chitosan/ glycerol decreases (Table 1). This may be taken as an indication of chitosan conformational changes within the film with the increasing of the plasticiser agent and shows the influence of polymer/ plasticiser content on the film's final structure.

\section{Conclusions}

The properties of films produced with film forming solutions (FFS) with 3 different concentrations of chitosan and 3 different levels of glycerol were measured.

It was observed that the rheological behaviour of the film forming solutions was dependent on chitosan concentration.
Consistency coefficient $(K)$ (and indirectly the molecular entanglement in the solution) affected the moisture content and the properties of the films obtained after drying. This may be due to differences on the drying behaviour during film production and thus significantly affecting the mechanical and thermal properties of the obtained films.

Glycerol addition caused changes in the films structure, by increasing free volume. This was reflected on the water and barrier, mechanical and thermal properties of the films. Moreover, glycerol affected the crystalline lattice of the film, by changing the $\mathrm{H}$-bonds in chitosan crystals. This conclusion is also supported by the FTIR results, were different interaction groups were observed according with the chitosan/ glycerol ratios.

The effect of polysaccharide/ plasticiser concentration on the microstructure and molecular dynamics of polymeric films systems will be a complementary study for understanding the behaviour of these structures.

Acknowledgments This work was supported by National Funds from FCT - Fundação para a Ciência e a Tecnologia, through project PEst-OE/ EQB/LA0016/2011.

Authors Joana F. Fundo, Andrea C. Galvis-Sánchez and Mafalda A. C. Quintas acknowledge FCT for research grants SFRH/ BD / 62176 / 2009, SFRH/BPD/37890/2007 and SFRH / BPD / 41715 / 2007, respectively.

\section{References}

1. B.L. Butler, P.J. Vergano, R.F. Testin, J.M. Bunn, J.L. Wiles, J. Food Sci. 61(5), 953-956 (1996)

2. A. Lazaridou, C.G. Biliaderis, Carbohydr. Polym. 48, 179-190 (2002)

3. P.C. Srinivasa, M.N. Ramesh, R.N. Tharanathan, Food Hydrocoll. 21, 1113-1122 (2007)

4. M. G. A. Vieira, M. A. da Silva, L. Oliveira dos Santos, M. M. Beppu, Eur. Polym. J. 47, 254-263 (2011)

5. J.D. Ferry, Viscoelastic Properties of Polymers, 3rd edn. (Wiley, New York, 1980)

6. V. Epure, M. Griffon, E. Pollet, L. Avérous, Carbohydr. Polym. 83, 947-952 (2011)

7. X. Zhang, M.D.D. Iko Burgar, E. Lourbakos, Biomacromolecules 6, 1661-1671 (2005)

8. I. Quijada-Garrido, V. Iglesias-Gonza'lez, J.M. Mazo'nArechederra, J.M. Barrales-Rienda, Carbohydr. Polym. 68, 173186 (2007)

9. Y. Roos, J. Food Eng. 24, 339-360 (1995)

10. C.G.T. Neto, J.A. Giacometti, A.E. Job, F.C. Ferreira, J.L.C. Fonseca, M.R. Pereira, Carbohydr. Polym. 62, 97-103 (2005)

11. M. Hasegawa, A. Isogai, F. Onabe, M. Usuda, R.H. Atalla, J. Appl. Polym. Sci. 45(11), 1873-1879 (1992)

12. C.K.S. Pillai, W. Paul, C.P. Sharma, Prog. Polym. Sci. 34, 641-678 (2009)

13. M. Rinaudo, Prog. Polym. Sci. 31, 603-632 (2006)

14. C.-H. Chen, F.-Y. Wang, C.-F. Mao, W.-T. Liao, C.-D. Hsieh, Int. J. Biol. Macromol. 43, 37-42 (2008)

15. N.E. Suyatma, L. Tighzert, A. Copinet, J. Agric. Food Chem. 53, 3950-3957 (2005)

16. L. Slade, H. Levine, Crit. Rev. Food Sci. Nutr. 30(2-3), 115-360 (1991) 
17. G. Dlubek, A.S. Gupta, J. Pionteckc et al., Polymer 46, 6075-6089 (2005)

18. T.H. McHugh, R. Avena-Bustillos, J.M. Krochta, J. Food Sci. 58(4), 899-903 (1993)

19. B.B.V. Guillard, C. Bonazzi, S. Guilbert, N. Gontard, J. Food Sci. 68(7), 2267-2277 (2003)

20. A.I. Bourbon, A.C. Pinheiro, M.A. Cerqueira et al., J. Food Eng. 106, 111-118 (2011)

21. J.T. Martins, M.A. Cerqueira, B.W.S. Souza, M.D.C. Avides, A.A. Vicente, J. Agric. Food Chem. 58, 1884-1891 (2010)

22. P.J.A. Sobral, F.C. Menegalli, M.D. Hubinger, M.A. Roques, Food Hydrocoll. 15, 423-432 (2001)

23. A. Barros. (Institut National Agronomique Paris-Grignon Paris, France, 1999)

24. J.F. Steffe, Rheological Methods in Food Process Engineering, 2nd edn. (Freeman Press, East Lansing, 1996)

25. M.A. Garcia, A. Pinotti, M.N. Martino, N.E. Zaritzky, Carbohydr. Polym. 56, 339-345 (2004)

26. S. Chillo, S. Flores, M. Mastromatteo, A. Conte, L. Gerschenson, M.A.D. Nobile, J. Food Eng. 88, 159-168 (2008)

27. T. Bourtoom, Int. Food Res. J. 15(2), 167-180 (2008)
28. B. Cuq, N. Gontard, J.-L. Cuq, S. Guilbert, J. Agric. Food Chem. 45, 622-626 (1997)

29. M. Lavorgna, F. Piscitelli, P. Mangiacapra, G.G. Buonocore, Carbohydr. Polym. 82, 291-298 (2010)

30. K. Ziani, J. Oses, V. Coma, J.I. Maté, LWT Food Sci. Technol. 41(10), 2159-2165 (2008)

31. D. Lourdin, H. Bizot, P. Colonna, J. Appl. Polym. Sci. 63, 10471053 (1997)

32. G. Roudaut, D. Simatos, D. Champion, E. Contreras-Lopez, M.L. Meste, Innovative Food Sci. Emerg. Technol. 5, 127-134 (2004)

33. S. Rivero, M.A. García, A. Pinotti, Innovative Food Sci. Emerg. Technol. 11, 369-375 (2010)

34. I.S. Arvanitoyannisa, A. Nakayamab, S.-i. Aibab, Carbohydr. Polym. 37, 371-382 (1998)

35. C.-A. Dai, Y.-F. Chen, M.-W. Liu, J. Appl. Polym. Sci. 99, 17951801 (2006)

36. K. Okuyama, K. Noguchi, T. Miyazawa, Macromolecules 30, 5849-5855 (1997)

37. C. Gao, M. Stading, N. Wellner et al., J. Agric. Food Chem. 54, 4611-4616 (2006)

38. G. Lawrie, I. Keen, B. Drew et al., Biomacromolecules 8, 2533 2541 (2007) 